PROCEEDINGS OF THE

AMERICAN MATHEMATICAL SOCIETY

Volume 126, Number 11, November 1998, Pages 3161-3168

S $0002-9939(98) 04477-3$

\title{
A NOTE ON COMPLETE INTERSECTIONS OF HEIGHT THREE
}

\author{
JUNZO WATANABE
}

(Communicated by Wolmer V. Vasconcelos)

\begin{abstract}
Let $k$ be a field of characteristic 0. If $I \subset k[x, y, z]$ is a complete intersection generated by three homogeneous elements of degrees $d_{1}, d_{2}, d_{3}$ with $2 \leq d_{1} \leq d_{2} \leq d_{3}$, then the reduction of $I$ by a general linear form is minimally generated by three elements if and only if $d_{3} \leq d_{1}+d_{2}-2$.
\end{abstract}

\section{INTRODUCTION}

This paper has grown out of a desire to prove the weak Lefschetz condition for Artinian complete intersections, homogeneous, of codimension three over a field of characteristic 0. The Main Theorem below is the most general result so far obtained in this direction. Although it may not be obvious, one realizes easily that it is a weaker statement than the weak Lefschetz condition; in other words any counter-example to the Main Theorem would have been a counter-example to the weak Lefschetz condition (see $\operatorname{Remark}(2)$ ).

The Main Theorem itself readily proves the weak Lefschetz condition for complete intersections of small degrees (Corollary 1) as well as for those in which one of the degrees is sufficiently greater than the others (Corollary 2).

Corollary 3 is another consequence of the Main Theorem which exhibits a set of minimal generators of the ideal $\left(f_{1}, f_{2}, f_{3}\right): z$ for a general element $z$.

Throughout this paper we work with polynomial rings (in at most three variables) over a field and ideals and elements that are homogeneous. Mostly the variables have degree 1 but at times it is necessary to consider a variable having degree 0 , which plays an important role.

The meaning of the words "generic linear form" should be self-explanatory, but for completeness we give a precise definition (Definition). For details we refer to [6, Appendix]. Generic variables are a set of generic linear forms such that their coefficients are algebraically independent over some field in consideration. Let $A=\bigoplus A_{i}$ be a graded Artinian algebra over a field $k=A_{0}$. Then we say that it has the weak Lefschetz condition, if there is an element $\ell$ of degree 1 such that the multiplication $\ell: A_{i} \rightarrow A_{i+1}$ is either injective or surjective for all $i$ (cf. [5]). If $I$ is a Gorenstein ideal of finite colength, it is well known that $I: f$ for any element

Received by the editors July 11, 1996 and, in revised form, March 28, 1997.

1991 Mathematics Subject Classification. Primary 13H05.

Key words and phrases. Homogeneous Artinian algebra, complete intersection, weak Lefschetz condition.

This research was supported by Project C of Tokai University.

(C)1998 American Mathematical Society 
$f \in A$ is a Gorenstein ideal (cf. [7, Lemma 4]). The letter $\mu$ denotes the number of generators.

\section{The Main Result And its CONSEQUences}

Definition. Let $R=\bigoplus_{i \geq 0} R_{i}$ be a graded ring, where $k=R_{0}$ is a field, and $R=$ $k\left[R_{1}\right]$. Suppose that $R_{1}$ is spanned by $x_{1}, x_{2}, \cdots, x_{n}$. Let $\xi_{1}, \cdots, \xi_{n}$ be algebraically independent elements over $R$. Let $k^{*}=k\left(\xi_{1}, \cdots, \xi_{n}\right)$ be the rational function field and put $R^{*}=R \otimes_{k} k^{*}$. Then we call the element $L=\xi_{1} x_{1}+\cdots+\xi_{n} x_{n} \in R^{*}$ a generic linear form for $R$. Unless otherwise specified we treat it as though it were an element of $R$, since in most cases a sufficiently general linear form in $R$ serves as "a generic linear form" ( cf. [6, Appendix]). The significance of $L$ is that it is generic for $R / I$ for whatever ideal $I \subset R$. We say that an element $g \in R_{1}$ is a general linear form if it plays the same role as a generic linear form, depending on a particular situation for a certain purpose.

Our main result is stated as follows.

Main Theorem. Let $R=k[x, y, z]$ be the polynomial ring over a field $k$ of characteristic 0 . Let $I$ be a complete intersection ideal of $R$ generated by homogeneous elements $f_{1}, f_{2}, f_{3} \in R$ of degrees $d_{1}, d_{2}, d_{3}$ respectively, where we assume that $2 \leq d_{1} \leq d_{2} \leq d_{3}$. Then the following conditions are equivalent.

(i) $\mu(I+\ell R / \ell R)=3$ for a generic linear form $\ell \in R$.

(ii) $d_{3} \leq d_{1}+d_{2}-2$.

Proof. (i) $\Rightarrow$ (ii). Let $\ell$ be a generic linear form. Then $f_{1}, f_{2}, \ell$ is a regular sequence. The socle degree of the ring $R /\left(f_{1}, f_{2}, \ell\right)$ is $d_{1}+d_{2}-2$. Hence if (ii) is not true, then $f_{3}$ is contained in the ideal $\left(f_{1}, f_{2}\right) R \bmod \ell R$, contradicting (i).

Before we start our proof for the other implication we prepare two easy lemmas.

Lemma 1. Let $R$ be a non-negatively graded ring over a local ring $R_{0}$ and let $M$ denote the unique homogeneous maximal ideal of $R$. Let $I \subset R$ be a homogeneous ideal. Suppose that $\ell \in M$ is a non-zero-divisor of $R / I$. Then $\mu(I+(\ell) /(\ell))=\mu(I)$.

Proof. First of all we note that Nakayama's lemma holds. I.e., a pre-image of a basis for $I / M I$ over $R / M$ generates $I$ as an ideal. By "“" we denote the reduction by $\ell$. Let $\left\{f_{1}, \cdots, f_{n}\right\}$ be a minimal generating set of $I$. It suffices to show that $\left\{\bar{f}_{1}, \cdots, \bar{f}_{n}\right\}$ is a minimal generating set of $\bar{I}$. Suppose not. Then, by Nakayama's lemma, one of the generators, say $\bar{f}_{1}$, is superfluous. I.e., $\bar{f}_{1}=\sum_{i=2}^{n} \bar{a}_{i} \bar{f}_{i}, \exists a_{i} \in R$. This means that $\sum_{i=1}^{n} a_{i} f_{i}=x \ell$, for $\exists x \in R$ with $a_{1}=-1$. Since $\ell$ is a non-zerodivisor of $R / I$ it follow that $x \in I$. Now write $x=\sum_{i=1}^{n} b_{i} f_{i}, \exists b_{i} \in R$ and we get $\left(1+\ell b_{1}\right) f_{1}=\sum_{i=2}^{n}\left(a_{i}-b_{i} \ell\right) f_{i}$. By comparing the homogenous parts of this equation in the smallest degree one obtains $f_{1}$ as a linear combination of $f_{2}, \cdots, f_{n}$, which is a contradiction.

Lemma 2. Let $k$ be a field of characteristic 0 and $R=k\left[x_{1}, \cdots, x_{n}\right]$ the polynomial ring over $k$. Suppose that $S \subset R_{1}$ consists of distinct linear forms as many as $\operatorname{dim} R_{d}$. Then the set $\left\{f^{d} \mid f \in S\right\}$ spans $R_{d}$. Here the word distinct means that none is a multiple of another.

Proof. Let $n=2$. Then the assertion is proved easily using the Van der Monde determinant. The general case follows by induction on $n$. (For a more general result see [2, Appendix III]. Also see [4, Lemma $3.1 \&$ Cor. 3.2].) 
Proof for $($ ii $) \Rightarrow$ (i) of Main Theorem. We may apply a general linear transformation of the variables, so we assume that $x, y, z$ are generic variables. Let $\alpha$ be a new variable with $\operatorname{deg} \alpha=0$ and put $\hat{R}=k[\alpha][x, y]$, which is the polynomial ring in $x, y$ with coefficients in $k[\alpha]$. Let $g$ be a general linear combination of $x$ and $y$ with coefficients in $k$ (so $g$ is a general element of $k[x, y]$ ) and consider the map $\phi: R \rightarrow \hat{R}$ defined by $x \mapsto x, y \mapsto y, z \mapsto g \alpha$. Note that $\phi$ is a graded homomorphism. Put $\hat{f}_{i}=\phi\left(f_{i}\right), \hat{I}=\left(\hat{f}_{1}, \hat{f}_{2}, \hat{f}_{3}\right) \hat{R}$. We will write $f_{i}=f_{i}(z) \in k[x, y][z]$ for $i=1,2,3$ and regard them as polynomials in $z$ with coefficients in $k[x, y]$. Then

$$
\hat{f}_{i}=f_{i}(g \alpha), \quad i=1,2,3 .
$$

By way of contradiction we assume that (i) in the statement of the Main Theorem is not true. Our first objective is to derive the inclusion

$$
f_{3}(g \alpha) \in\left(f_{1}(g \alpha), f_{2}(g \alpha)\right)
$$

in the ring $k[\alpha]_{(\alpha)}[x, y]$, which is the polynomial ring in $x, y$ over the discrete valuation ring $k[\alpha]_{(\alpha)}$. The failure of (i) means that $\mu(I+(\ell) /(\ell))=2$ for all generic linear forms, and in this case the ideal $I+(\ell) /(\ell)$ is generated by $f_{1}$ and $f_{2} \bmod (\ell)$ due to the degree reason. Notice that, for any $\alpha_{0} \in k$, we have the isomorphism

$$
\hat{R} / \hat{I}+\left(\alpha-\alpha_{0}\right) \hat{R} \cong R /\left(I+\left(\alpha_{0} g-z\right)\right) R .
$$

The element $\alpha_{0} g-z$ is a generic linear form for $R / I$ for all but a finite number of values of $\alpha_{0}$, and particularly it is generic for $\alpha_{0}=0$ since $z$ is a generic variable. It follows that the map $k[\alpha] \rightarrow \hat{R} / \hat{I}$ is locally flat at $\alpha=0$ because the two fibers have the same dimension (which is $d_{1} d_{2}$ ). Thus $\alpha$ is a non-zero-divisor for $\hat{I} \hat{R} \otimes_{k[\alpha]} k[\alpha]_{(\alpha)}$. Hence it is possible to apply Lemma 1 to conclude that $\mu(\hat{I})=2$. Thus we have derived the inclusion (1) from the assumption that the statement (i) of the Main Theorem is not true. Now we write:

$$
f_{3}(g \alpha)=\sum_{i=1}^{2} A_{i}(\alpha) f_{i}(g \alpha)
$$

where $A_{i}(\alpha) \in k[\alpha]_{(\alpha)}[x, y]$ are homogeneous elements. The notation $A_{i}(\alpha)$ is used to mean that they are treated as functions in $\alpha$. Let us write the same equation as (2) for another general element $h$ of $k[x, y]$ with $B_{i}(\alpha)$ in place of $A_{i}(\alpha)$. Namely,

$$
f_{3}(h \alpha)=\sum_{i=1}^{2} B_{i}(\alpha) f_{i}(h \alpha) .
$$

We are going to prove the following two statements (I) and (II).

(I) For any $n \geq 0, A_{i}^{(n)}(0)$ is divisible by $g^{n}$ in the ring $k[x, y]$, for $i=1,2$.

(II) For any $n \geq 0, A_{i}^{(n)}(0) / g^{n}=B_{i}^{(n)}(0) / h^{n}, i=1,2$.

Here we have written $A_{i}^{\prime}(\alpha)=\frac{d}{d \alpha} A_{i}(\alpha)$. Note that $A_{i}^{(n)}(0) \in k[x, y]$ since $A_{i}(\alpha) \in$ $k[\alpha]_{(\alpha)}[x, y]$. Once (I) is proved, it implies, among other things, that $A_{i}^{(n)}(0)=0$ for all sufficiently large $n$ as the differentiation by $\alpha$ does not change the degree with respect to $x, y$. Taking the Taylor expansion of $A_{i}(\alpha)$ in powers of $\alpha$ and substituting $\alpha=z / g$ in the equation (2), (I) further implies that

$$
f_{3}(z) \in\left(f_{1}(z), f_{2}(z)\right) R
$$


which contradicts the assumption ht $\left(f_{1}, f_{2}, f_{3}\right)=3$. Thus it suffices to prove (I) to complete the proof of the Main Theorem. We note that the statement (II) is necessary as an inductive set up for a proof of (I).

Proof of (I) and (II). We proceed by induction on $n$. Let $n=0$. (I) is trivial. We prove (II). Set $\alpha=0$ in the equations (2) and (3) and make the difference. We get:

$$
0=\sum_{i=1}^{2}\left\{A_{i}(0)-B_{i}(0)\right\} f_{i}(0) \text {. }
$$

From (2) and (3) follows $\operatorname{deg} A_{i}(0)=\operatorname{deg} B_{i}(0)=d_{3}-d_{i}$ for $i=1,2$. Hence the degree of the RHS of the equation (4) is $d_{3}$. Recall that we are using generic variables. Hence $f_{1}(0)$ and $f_{2}(0)$ are coprime and they can have only a Koszul relation whose degree is $d_{1}+d_{2}$. Since $d_{3} \leq d_{1}+d_{2}-2<d_{1}+d_{2}$ it is possible only if $A_{i}(0)=B_{i}(0)$ for $i=1,2$. This is (II) in the case $n=0$.

Now assuming the induction hypothesis we prove (I) and (II) for $n$. We prove (I) first. Differentiate the equation (2) $n$ times with respect to $\alpha$. We get:

$$
f_{3}^{(n)}(g \alpha) g^{n}=\sum_{i=1}^{2} \sum_{j=0}^{n}\left(\begin{array}{c}
n \\
j
\end{array}\right) A_{i}^{(n-j)}(\alpha) f_{i}^{(j)}(g \alpha) g^{j} .
$$

(We are writing $f_{i}^{\prime}(z)=\frac{d}{d z} f_{i}(z)$, and $A_{i}^{\prime}(\alpha)=\frac{d}{d \alpha} A_{i}(\alpha)$.) In the equation (5) set $\alpha=0$, and rewrite it as

$$
f_{3}^{(n)}(0) g^{n}-\sum_{i=1}^{2} \sum_{j=1}^{n}\left(\begin{array}{c}
n \\
j
\end{array}\right) A_{i}^{(n-j)}(0) f_{i}^{(j)}(0) g^{j}=\sum_{i=1}^{2} A_{i}^{(n)}(0) f_{i}^{(0)}(0) .
$$

The induction hypothesis (I) implies that the LHS of (6) is divisible by $g^{n}$. Hence we have that

$$
f_{3}^{(n)}(0)-\sum_{i=1}^{2} \sum_{j=1}^{n}\left(\begin{array}{c}
n \\
j
\end{array}\right) \frac{1}{g^{n-j}} A_{i}^{(n-j)}(0) f_{i}^{(j)}(0) \in\left(f_{1}(0), f_{2}(0)\right): g^{n} .
$$

The induction hypothesis (II) implies that the LHS of the equation (7) is also contained in the ideal $\left(f_{1}(0), f_{2}(0)\right): h^{n}$. Choosing various $g$ 's it follows that the LHS of $(7)$ is in fact contained in $\left(f_{1}(0), f_{2}(0)\right):(x, y)^{n}$ by Lemma 2 . By the duality of a Gorenstein algebra we have:

$$
\left(f_{1}(0), f_{2}(0)\right):(x, y)^{n}=\left(f_{1}(0), f_{2}(0)\right)+(x, y)^{d_{1}+d_{2}-1-n} .
$$

But the degree of the LHS of the equation (7) is $d_{3}-n$ which is strictly smaller than $d_{1}+d_{2}-1-n$. This implies that the LHS of $(7)$ is in fact in the ideal $\left(f_{1}(0), f_{2}(0)\right)$. Thus the LHS of the equation (6) is an element of $g^{n}\left(f_{1}(0), f_{2}(0)\right)$. This means that the RHS of the equation (6) can be written as

$$
\sum_{i=1}^{2} A_{i}^{(n)}(0) f_{i}^{(0)}(0)=\sum_{i=1}^{2} g^{n} P_{i} f_{i}(0),
$$

with suitable homogeneous elements $P_{i} \in k[x, y]$. Note that the degree of $A_{i}^{(n)}(0)$ is $d_{3}-d_{i}$ which is automatically the degree of $g^{n} P_{i}$. It follows that $A_{i}^{(n)}(0)=g^{n} P_{i}$ for $i=1,2$ because there is not a syzygy of $f_{1}(0), f_{2}(0)$ with degree less than $d_{1}+d_{2}$. 
Thus we have proved (I). To prove (II) we have to show that $P_{1}$ and $P_{2}$ do not depend on $g$. From the equation (6), we have

$$
\sum_{i=1}^{2} \frac{1}{g^{n}} A_{i}^{(n)}(0) f_{i}(0)=f_{3}^{(n)}(0)-\sum_{i=1}^{2} \sum_{j=1}^{n} \frac{1}{g^{n-j}} A_{i}^{(n-j)}(0) f_{i}^{(j)}(0) .
$$

The same formula can be derived from the equation (3), but the induction hypothesis (II) implies that the right-hand side of this equation is independent of $g$. This means that

$$
\sum_{i=1}^{2} \frac{1}{g^{n}} A_{i}^{(n)}(0) f_{i}(0)=\sum_{i=1}^{2} \frac{1}{h^{n}} B_{i}^{(n)}(0) f_{i}(0) .
$$

Again by the degree reason we have

$$
\frac{1}{g^{n}} A_{i}^{(n)}(0)=\frac{1}{h^{n}} B_{i}^{(n)}(0) \text { for } i=1,2 .
$$

This is (II). Now the proof is complete.

Remark. (1) In the same notation as in the Main Theorem if $d_{3}>d_{1}+d_{2}-2$ then the weak Lefschetz condition holds on the ring $R / I$. In fact the multiplication by a linear form on $R / I$ acts in the same way as it does on $R /\left(f_{1}, f_{2}\right)$ up to the middle of the graded pieces. For the other half we can use the duality. Hence the multiplication by a general element is piece-wise full rank since depth $R /\left(f_{1}, f_{2}\right)=$ 1.

(2) Any counter-example to the Main Theorem would have been be a counterexample to the weak Lefschetz condition for $R / I$. In fact consider the exact sequence:

$$
0 \rightarrow R / I: \ell \rightarrow R / I \rightarrow R / I+(\ell) \rightarrow 0
$$

where the map on the left is the multiplication by $\ell$. If the weak Lefschetz condition holds on the ring $R / I$ then the Hilbert function of the ring $R / I+(\ell)$ is the first difference of the Hilbert function of $R / I$ (with non-positive part ignored). On the other hand if $\mu(I+(\ell) /(\ell))=2$ then the Hilbert function of $R / I+(\ell)$ is symmetric. It is not difficult to see that this is possible if and only if $d_{3}>d_{1}+d_{2}-2$.

(3) Assume that $d_{3} \leq d_{1}+d_{2}-2$. Denote by "" the reduction by a general linear form. Let

$$
0 \rightarrow \bigoplus_{i=1}^{2} \bar{R}\left(-e_{i}\right) \rightarrow \bigoplus_{i=1}^{3} \bar{R}\left(-d_{i}\right) \rightarrow \bar{I}
$$

be a minimal free resolution of $\bar{I}$. Then the weak Lefschetz condition holds on $R / I$ if and only if $\left|e_{1}-e_{2}\right|=0$ or 1 according as $\sum d_{i}$ is even or odd, since in this case the Hilbert function of the ring $\bar{R} /\left(\bar{f}_{1}, \bar{f}_{2}, \bar{f}_{3}\right)$ is the smallest with $d_{1}, d_{2}, d_{3}$ fixed.

Corollary 1. Let $I=\left(f_{1}, f_{2}, f_{3}\right) \subset R$ be a regular sequence with degrees $d_{1}, d_{2}, d_{3}$. If $d_{i} \leq 3, \forall i$, then the weak Lefschetz condition holds on the ring $R / I$.

Proof. Consider the case $d_{i}=3, \forall i$. Let $\ell$ be any linear form satisfying $\mu(I+(\ell) /(\ell))=3$. Then we have a minimal free resolution of $\bar{R} / \bar{I}$ as follows:

$$
0 \rightarrow \bar{R}(-4) \bigoplus \bar{R}(-5) \rightarrow \bar{R}(-3)^{\oplus 3} \rightarrow \bar{I} \rightarrow 0
$$

Note that the generator degrees $(3,3,3)$ uniquely determine the relation degrees $(4,5)$ by the Hilbert-Burch theorem (cf. the proof of Corollary 3 (ii) below). It 
follows that the Hilbert function of $\bar{R} / \bar{I}$ is $(1,2,3,1)$, which implies that the Hilbert function of $R /(I: \ell)$ is $(1,3,6,6,3,1)$. This shows that the multiplication by $\ell$ on the ring $R / I$ has full rank at each graded piece. The cases $\left(d_{1}, d_{2}, d_{3}\right)=$ $(2,2,2)$ and $(2,3,3)$ are similar. For the case $(2,2,3)$ we can use Remark $(1)$. If one of $d_{i}$ 's is equal to 1 the strong Lefschetz condition holds by [3, Theorem 2.9].

Corollary 2. Let $I=\left(f_{1}, f_{2}, f_{3}\right) \subset R$ be a regular sequence with degrees $d_{1}, d_{2}, d_{3}$. Assume that $d_{3} \geq \operatorname{Max}\left\{d_{1}, d_{2}\right\}$. If $d_{3} \geq d_{1}+d_{2}-3$ then the Weak Lefschetz condition holds on the ring $R / I$.

Proof. (i) The case $d_{3}>d_{1}+d_{2}-2$ was explained in Remark (1).

(ii) Assume that $d_{3}=d_{1}+d_{2}-2$. Denote by "'” the reduction by a general element. One sees easily that $\bar{f}_{3}$ is a generator of the socle of $\bar{R} /\left(\bar{f}_{1}, \bar{f}_{2}\right)$. Hence we have $\bar{x} \bar{f}_{3} \in\left(\bar{f}_{1}, \bar{f}_{2}\right)$ and $\bar{y} \bar{f}_{3} \in\left(\bar{f}_{1}, \bar{f}_{2}\right)$, which gives two (independent) syzygies of the same degree. Thus by Remark (3) the weak Lefschetz condition follows.

(iii) Assume that $d_{3}=d_{1}+d_{2}-3$. We may assume that $\bar{f}_{1}, \bar{f}_{2}$ are a regular sequence. Then we have that $\bar{x} \bar{f}_{3}$ and $\bar{y} \bar{f}_{3}$ are linearly dependent modulo $\left(\bar{f}_{1}, \bar{f}_{2}\right)$ as they sit in the socle of $\bar{R} /\left(\bar{f}_{1}, \bar{f}_{2}\right)$. This gives a syzygy of degree $d_{3}+1$. The degree of another basic syzygy is automatically $d_{3}+2$. Hence by Remark (3) we have the weak Lefschetz condition.

Remark. (4) As before let $\left(d_{1}, d_{2}, d_{3}\right)$ be the generator degrees. Corollaries 1 and 2 imply in particular that if $d_{1}=d_{2}=3$, then for any $d_{3}>0$ the weak Lefschetz condition holds on the ring $R /\left(f_{1}, f_{2}, f_{3}\right)$. If $d_{1}=d_{2}=4$ then for any $d_{3}>0$ except for $d_{3}=4$ the weak Lefschetz condition holds. Thus the case where the generator degrees are $(4,4,4)$ is the first open case for the weak Lefschetz condition.

Corollary 3. Let $R=k[x, y, z]$ be the polynomial ring over a field $k$ of characteristic 0 . Let $I$ be a complete intersection ideal of $R$ generated by homogeneous elements $f_{1}, f_{2}, f_{3} \in R$ of degrees $d_{1}, d_{2}, d_{3}$ respectively, where we assume that $2 \leq d_{1} \leq d_{2} \leq d_{3}$. Let $\ell$ be a general linear form. Then we have

(i) $d_{3}>d_{1}+d_{2}-2 \Rightarrow I$ : $\ell$ is generated by 3 elements.

(ii) $d_{3} \leq d_{1}+d_{2}-2 \Rightarrow I: \ell$ is generated by 5 elements.

Proof. (i) Let $j$ be the smallest integer such that

$$
\operatorname{dim}(R / I)_{j}>\operatorname{dim}(R / I)_{j+1} .
$$

First we show that $d_{3}=j+1$. Notice that $\operatorname{dim}[R / I]_{i}=\operatorname{dim}\left[R /\left(f_{1}, f_{2}\right)\right]_{i}$ for $i=$ $0, \cdots, d_{3}-1$. Hence the dimensions are not decreasing up to $i=d_{3}-1$, because the $\operatorname{ring} R /\left(f_{1}, f_{2}\right)$ is a 1-dimensional CM algebra. On the other hand $\operatorname{dim}\left[R /\left(f_{1}, f_{2}\right)\right]_{i}$ reaches the maximum at $i=d_{1}+d_{2}-2$. Now the hypothesis implies that the Hilbert series of $R / I$ begins decreasing just at $i=d_{3}$ as claimed. Let $\ell$ be a general linear form and $f$ a non-zero element in $\operatorname{Ker}\left[\ell:(R / I)_{j} \rightarrow(R / I)_{j+1}\right]$. This means that $\ell f=a_{1} f_{1}+a_{2} f_{2}+a_{3} f_{3}$ with $a_{i} \in R$. By a degree reason $a_{3}$ is a non-zero constant. Thus we have

$$
\left(f_{1}, f_{2}, f_{3}\right): \ell \supseteq\left(f_{1}, f_{2}, f\right) \supset\left(f_{1}, f_{2}, f_{3}\right) .
$$

The socle degree of $R /\left(f_{1}, f_{2}, f_{3}\right): \ell$ is one less than that of $R /\left(f_{1}, f_{2}, f_{3}\right)$, since $\ell$ is of degree 1 . Thus $R /\left(f_{1}, f_{2}, f_{3}\right): \ell$ and $R /\left(f_{1}, f_{2}, f\right)$ have the same socle degree. By [7, Lemma 4], there is an element $x \in R$ such that $\left(f_{1}, f_{2}, f_{3}\right): \ell=\left(f_{1}, f_{2}, f\right): x$. If $x$ were not a unit, the socle degree of $\left(f_{1}, f_{2}, f\right): x$ would be strictly less than the socle 
degree of $\left(f_{1}, f_{2}, f\right)$. Thus $x$ is in fact a unit and we have $\left(f_{1}, f_{2}, f_{3}\right): \ell=\left(f_{1}, f_{2}, f\right)$. This completes the proof.

(ii) We may assume that $z$ is a general element for $I$. We write $f_{i}=f_{i}(z)$ and regard them as polynomials in $z$ with coefficients in $k[x, y]$. Further write $f_{i}=$ $f_{i}(0)+h_{i} z$ where $h_{i} \in R$ are homogeneous elements of degree $d_{i}-1$. Let $M=\left(a_{i j}\right)$ be a minimal syzygy matrix of the vector ${ }^{t}\left(f_{1}(0), f_{2}(0), f_{3}(0)\right) \subset k[x, y]^{\oplus 3}$ over the ring $k[x, y]$. By the Main Theorem it is a 2 by 3 matrix with homogeneous forms of positive degrees as entries. The Hilbert-Burch theorem says that $\left(f_{1}, f_{2}, f_{3}\right)=$ $c\left(\Delta_{1},-\Delta_{2}, \Delta_{3}\right), \exists c \in k$, where $\Delta_{i}$ is the determinant of the matrix $M$ with $i$ th column deleted. Define the matrix $P$ by:

$$
P=\left(\begin{array}{ccccc}
0 & -h_{3} & -h_{2} & -a_{11} & -a_{21} \\
h_{3} & 0 & -h_{1} & -a_{12} & -a_{22} \\
h_{2} & h_{1} & 0 & -a_{13} & -a_{23} \\
a_{11} & a_{12} & a_{13} & 0 & -z \\
a_{21} & a_{22} & a_{23} & z & 0
\end{array}\right)
$$

Let $J$ be the ideal generated by the Pfaffians of $P$. As a consequence of the HilbertBurch theorem, the three of the Pfaffians are $\left\{f_{1}, f_{2}, f_{3}\right\}$. The other two are :

$$
\begin{aligned}
& f_{4}=h_{1} a_{11}-h_{2} a_{12}+h_{3} a_{13}, \\
& f_{5}=h_{1} a_{21}-h_{2} a_{22}+h_{3} a_{23} .
\end{aligned}
$$

Since $J \supset I$, grade $J=3$. By the structure theorem of height three Gorenstein ideals (cf. [1]), one sees that $J$ is a Gorenstein ideal minimally generated by these five elements. The structure theorem also says that $P$ is a syzygy matrix of ${ }^{t}\left(f_{1}, \cdots, f_{5}\right)$. Hence we have

$$
\left(f_{1}, f_{2}, f_{3}\right): z \supseteq J \supset\left(f_{1}, f_{2}, f_{3}\right) .
$$

By the same argument for (i) we see that the socle degree of $R /\left(f_{1}, f_{2}, f_{3}\right): z$ is one less than that of $R /\left(f_{1}, f_{2}, f_{3}\right)$ and hence no Gorenstein ideal can exist properly between $\left(f_{1}, f_{2}, f_{3}\right): z$ and $\left(f_{1}, f_{2}, f_{3}\right)$. Thus we have $\left(f_{1}, f_{2}, f_{3}\right): z=$ $\left(f_{1}, f_{2}, f_{3}, f_{4}, f_{5}\right)$, which completes the proof.

\section{ACKNowledgments}

The author would like to express his deep thanks to Professor Anthony Iarrobino, Mats Boij and David Eisenbud for many discussions of the subject. Also he would like to thank the referee for a critical reading of the original manuscript of this paper. Finally the author would like to thank the Mathematics Department of Northeastern University for its hospitality for ten months which made this research possible.

\section{REFERENCES}

[1] D. A. Buchsbaum and D. Eisenbud, Algebraic structures for finite free resolutions, and some structure theorem for ideals of codimension 3, Amer. J. Math. 99 (1977), 447-485. MR $\mathbf{5 6 : 1 1 9 8 3}$

[2] J. H. Grace and A. Young, The algebra of invariants, Cambridge Univ. Press, 1903.

[3] A. Iarrobino, Associated graded algebra of a Gorenstein algebra, Mem. Amer. Math. Soc. 107 1994. MR 94f: 13009

[4] _ Compressed algebras, Trans. Amer. Math. Soc. 285 (1984), 337-378. MR 85j:13030

[5] J. Watanabe, The Dilworth number of Artinian rings and finite posets with rank function, Adv. Stud. Pure Math. 11 (1987), 303-312. MR 89k:13015 
[6] _

[7] _ A note on Gorenstein rings of embedding codimension three, Nagoya Math. J. 50 (1973), 227-232. MR 47:8526

Department of Mathematical Sciences, Tokai University, Hiratsuka 259-1292, Japan

E-mail address: junzowat@ss.u-tokai.ac.jp 